\title{
Generalized Plasma Dispersion Function: One-Solve-All Treatment, Visualizations, and Application to Landau Damping
}

\author{
Hua-sheng XIE (谢华生) ${ }^{1, \text { * }}$ \\ ${ }^{1}$ Institute for Fusion Theory and Simulation, Zhejiang University, Hangzhou, 310027, PRC
}

(Dated: September 10, 2013)

\begin{abstract}
A unified, fast, and effective approach is developed for numerical calculation of the well-known plasma dispersion function with extensions from Maxwellian distribution to almost arbitrary distribution functions, such as the $\delta$, flat top, triangular, $\kappa$ or Lorentzian, slowing down, and incomplete Maxwellian distributions. The singularity and analytic continuation problems are also solved generally. Given that the usual conclusion $\gamma \propto \partial f_{0} / \partial v$ is only a rough approximation when discussing the distribution function effects on Landau damping, this approach provides a useful tool for rigorous calculations of the linear wave and instability properties of plasma for general distribution functions. The results are also verified via a linear initial value simulation approach. Intuitive visualizations of the generalized plasma dispersion function are also provided.
\end{abstract}

\section{INTRODUCTION}

In a one-dimensional, one-species, non-relativistic electrostatic plasma system, the Langmuir wave dispersion relation is [1]

$$
D(k, \omega)=1-\frac{\omega_{p}^{2}}{k^{2}} \int_{C} \frac{\partial f_{0} / \partial v}{v-\omega / k} d v=0,
$$

where $k$ is the wave vector, $\omega=\omega_{r}+i \gamma$ is the frequency, $\omega_{p}=\sqrt{4 \pi n_{0} q^{2} / m}$ is the plasma frequency and $C$ is the Landau integral contour.

For Maxwellian distribution $f_{0}=F_{M}$, with

$$
F_{M}(v)=\frac{1}{\sqrt{\pi} v_{t}} e^{-\frac{v^{2}}{v_{t}^{2}}}
$$

the well-known plasma dispersion function (PDF)

$$
Z_{M}(\zeta)=\frac{1}{\sqrt{\pi}} \int_{-\infty}^{\infty} \frac{e^{-z^{2}}}{z-\zeta} d z, \quad \Im(\zeta)>0,
$$

with analytic continuation to $\Im(\zeta) \leq 0$, is defined by Fried and Conte[2], where $\zeta=\omega /\left(k v_{t}\right)$ and $z=v / v_{t}$. Hence, (1) is rewritten to

$$
D(k, \omega)=1-\frac{1}{\left(k \lambda_{D}\right)^{2}} \frac{1}{2} Z_{M}^{\prime}(\zeta)=0,
$$

with also

$$
Z_{M}^{\prime}(\zeta)=-2\left[1+\zeta Z_{M}(\zeta)\right],
$$

where $\lambda_{D}=\sqrt{T / m} / \omega_{p}$ and $v_{t}=\sqrt{2 T / m}$. A $\sqrt{2}$ difference in the normalizations between $v_{t}$ and $T$ should be noted.

Analytic properties and numerical approaches for the usual PDF (3), which is similarly related to complex error function, Faddeeva function, or Dawson integral, have

*Electronic mail: huashengxie@gmail.com been extensively studied since Fried and Conte 2]. Good $Z_{M}(\zeta)$ function numerical schemes for practical application can also be easily found. However, in studying other distribution functions, it should be treated separately. The singularity in real line and analytic continuation to $\Im(\zeta) \leq 0$ usually requires careful treatment, otherwise it would be confusing and would yield incorrect results.

A family of distributions, i.e., $\kappa$ distributions or generalized Lorentzian distributions $\underline{3}$

$$
F_{\kappa}=A_{\kappa}\left[1+\frac{1}{\kappa} \frac{v^{2}}{v_{t}^{2}}\right]^{-\kappa},
$$

with the normalization constant

$$
A_{\kappa}=\frac{1}{v_{t}} \frac{\Gamma(\kappa)}{\Gamma(\kappa-1 / 2)} \frac{1}{\sqrt{\pi \kappa}},
$$

are very useful for space and astrophysical plasma and have been studied intensively since Summers and Thorne 4, where $\Gamma$ is the Euler gamma function. Recently, Baalrud 5 also investigated a semi-infinite integral for Maxwellian distribution, called the incomplete PDF.

Each author uses his or her own technique to treat the Landau contour. For instance, Baalrud [5] treated the incomplete PDF via direct numerical integral and continued fraction expansion, whereas Hellberg and Mace $\underline{6}$ treated the $\kappa$ distribution using Gauss hypergeometric function.

The generalized plasma dispersion function (GPDF) can be defined as

$$
Z(\zeta)=Z(\zeta, F)=\int_{C} \frac{F}{z-\zeta} d z,
$$

and its derivative

$$
Z_{p}(\zeta)=\int_{C} \frac{\partial F / \partial z}{z-\zeta} d z=Z^{\prime}(\zeta, F),
$$

with the original PDF as a special case when $F=$ $e^{-z^{2}} / \sqrt{\pi}$. Developing systematic methods to treat arbitrary physical reasonable distribution functions (e.g., 

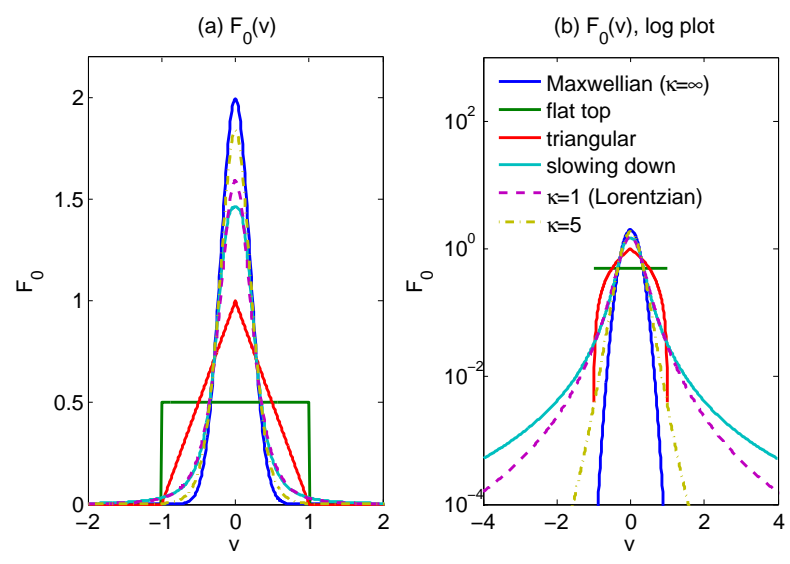

FIG. 1: Typical distribution functions.

$\left.f \geq 0, \int f d v<\infty\right)$ in one scheme, i.e., one-solve-all, is advantageous. For instance, several typical distribution functions are shown in Fig.1.

In this study, we investigate the analytical properties (particularly the singularity and analytic continuation problems) and develop a general numerical scheme for GPDF, i.e., for almost arbitrary input function $F$.

This problem was also discussed by Robinson [7, who used the linear combination of orthogonal functions. Three sets of orthogonal functions, Hermite, Legendre, and Chebyshev polynomials were discussed. Robinson's method is very similar to our treatment in this study. However, he has neither given systematic results of the analytic continuation nor developed a one-solve-all scheme for practical application.

Our approach, which is discussed in Sec III is based on Hilbert transform (HT) and fast Fourier transform (FFT). After solving GPDF generally, we show several visualizations of GPDF in Sec III. The distribution function effects on Landau damping are revisited in SecIV. and a summary and discussion are given in Sec $\mathrm{V}$.

\section{ONE-SOLVE-ALL SCHEME FOR GENERALIZED PLASMA DISPERSION FUNCTION}

\section{A. Hilbert transform and analytic continuation}

HT is defined as

$$
g(z)=H(f(z))=\frac{1}{\pi} \int_{-\infty}^{\infty} \frac{f\left(z^{\prime}\right)}{z^{\prime}-z} d z^{\prime},
$$

which can also be viewed as a convolution

$$
g(z)=\frac{1}{\pi z} * f(z)
$$

or the inverse

$$
f(z)=-\frac{1}{\pi z} * g(z)
$$

where $f(z)$ and $g(z)$ are called a Hilbert pair. HT usually represents a $90^{\circ}$ phase shift of input function.

Some useful properties include

1. $H\left(c_{1} f_{1}(z)+c_{2} f_{2}(z)\right)=c_{1} g_{1}(z)+c_{2} g_{2}(z)$,

2. $H(H(f(z)))=-f(z)$,

3. $H(f(z+a))=g(z+a)$,

4. $H(f(a z))=\operatorname{sgn}(a) g(a z)$,

5. $H\left(\frac{d^{n} f(z)}{d z^{n}}\right)=\frac{d^{n} g(z)}{d z^{n}}$,

6. $f(z)+i g(z)$ is an analytical function.

A good scheme for numerically calculating HT in real line is provided by Weideman [8]. Two other numerical methods are, using (10), i.e.,

$$
g(z) \simeq \frac{2}{\pi} \sum_{n=-\infty}^{\infty} \frac{f(z+(2 n+1) h)}{2 n+1}
$$

where $h$ is the step size, or using (11), i.e.,

$$
g(z)=i f t\left[f t\left(\frac{1}{\pi z}\right) \cdot f t(f(z))\right],
$$

where $f t()$ and ift() denote the Fourier transform and its inverse.

The methods in Weideman's 1995 paper 8 or via (13) and (14) are mainly for calculating integral principal value $(\mathrm{PV})$ in real line.

In fact, the definition of 100 is not a single function. For simplification, we require $f(z)$ be an entire function that is integrable in the range of $-\infty$ to $+\infty$. The plasma dispersion function is defined for $\Im(z)>0$, and thus, should be extended to $\Im(z) \leq 0$, which is [10]

$$
g^{+}(z)=\left\{\begin{array}{cc}
\frac{1}{\pi} \int_{-\infty}^{\infty} \frac{f\left(z^{\prime}\right)}{z^{\prime}-z} d z^{\prime}, & \Im(z)>0 \\
\frac{1}{\pi} P V \int_{-\infty}^{\infty} \frac{f\left(z^{\prime}\right)}{z^{\prime}-z} d z^{\prime}+i f(z), & \Im(z)=0 \\
\frac{1}{\pi} \int_{-\infty}^{\infty} \frac{f\left(z^{\prime}\right)}{z^{\prime}-z} d z^{\prime}+2 i f(z), & \Im(z)<0
\end{array}\right.
$$

For instance, the HT of Lorentzian distribution $f(z)=$ $\frac{a}{\pi} \frac{1}{z^{2}+a^{2}}$ is

$$
g(z)= \begin{cases}-\frac{1}{\pi} \frac{1}{(z+i a)}, & \Im(z)>0 \\ -\frac{z}{\left(z^{2}+a^{2}\right)}, & \Im(z)=0 \\ -\frac{1}{\pi} \frac{1}{(z-i a)}, & \Im(z)<0\end{cases}
$$

whereas

$$
g^{+}(z)=-\frac{1}{\pi} \frac{1}{(z+i a)},
$$

which is consistent with 15).

$g^{-}(z)$ can be defined in a similar manner if we want to extend a function from lower half plane to the entire complex plane.

Weideman 9] also provided a method to calculate $g^{+}(z)$ of the HT of Gaussian function in upper half plane, which is related to $\operatorname{PDF} Z_{M}(\zeta)$. 


\section{B. One-solve-all approach}

A comparison of (8) and (9) with $(15)$ indicates $Z=$ $\pi g^{+}$and $Z_{p}=\pi g^{\prime+}$ with $F=f$ and $F^{\prime}=f^{\prime}$. Thus, GPDF is merely a HT of distribution function and shares the same properties of HT as listed in the above subsection.

Jones et al. [10] also discussed the contour integral problem of GPDF and used transformation $z=\tan (t)$ to map the integral of $z \in(-\infty, \infty)$ to $t \in(-\pi, \pi)$. This method is merely an alteration of (13) and requires other tricks to avoid singularity. Moreover, the method is time consuming and not suitable for high accuracy calculation as the discrete step should be very small to avoid divergence. Another direct numerical integral result is shown by Guio et al. [11] with typical errors of $10^{-4}$.

In this paper, we use (15) to extend Weideman's approach [8, 9] to the entire complex plane, where the orthogonal functions is $e^{i \theta}$, which can be evaluated very rapidly by FFT, instead of the Hermite, Legendre, and Chebyshev polynomials used by Robinson 7 .

The key steps are summarized as follows.

Assuming an expansion

$$
[W(v)]^{-1} F(v)=\sum_{n=-\infty}^{\infty} a_{n} \rho_{n}(v), \quad v \in \mathcal{R}
$$

where $\left\{\rho_{n}(v)\right\}$ is an orthogonal basis set with weight function $W(v)$, i.e.,

$$
\int_{-\infty}^{\infty} W(v) \rho_{n}(v) \rho_{m}^{*}(v) d v=A \delta_{m, n}
$$

where the asterisk denotes complex conjugation and $\delta_{m, n}$ is the Kronechker delta. The coefficients are

$$
a_{n}=\frac{1}{A} \int_{-\infty}^{\infty} F(v) \rho_{n}^{*}(v) d v
$$

Then

$$
\frac{F(v)}{v-z}=\sum_{n=-\infty}^{\infty} a_{n}\left[W(v) \frac{\rho_{n}(v)}{v-z}\right]
$$

For the upper half plane, we use weight function $W(v)=1 /\left(L^{2}+v^{2}\right)[9]$ and basis functions

$$
\rho_{n}(v)=\frac{(L+i v)^{n}}{(L-i v)^{n}}
$$

which is a Fourier form because $e^{i \theta}=(L+i v) /(L-i v)$ with $v=L \tan (\theta / 2)$ and $d v / d \theta=\left(L^{2}+v^{2}\right) /(2 L)$, then $a_{n}$ can be evaluated using FFT and we can obtain $A=\pi / L$ using $\int_{-\pi}^{\pi} e^{i n \theta} e^{-i m \theta} d \theta=2 \pi \delta_{m, n}$.

Using residues, for $\Im(\zeta)>0$, we find the integrals

$$
\int_{-\infty}^{\infty} \frac{W(v)}{v-z} \frac{(L+i v)^{n}}{(L-i v)^{n}} d v=\left\{\begin{array}{cc}
\frac{i \pi}{L} \frac{1}{(L-i z)}, & n=0 \\
\frac{2 i \pi}{L^{2}+z^{2}} \frac{(L+i z)^{n}}{(L-i z)^{n}}, & n>0 \\
0, & n<0 .
\end{array}\right.
$$

We obtain

$g^{+}(z)=\frac{2 i}{L^{2}+z^{2}} \sum_{n=1}^{\infty} a_{n}\left(\frac{L+i z}{L-i z}\right)^{n}+\frac{i a_{0}}{L(L-i z)}, \quad \Im(z)>0$

For the lower half plane, $\Im(\zeta)<0$, we use $F(z)=$ $\left[F\left(z^{*}\right)\right]^{*}$ and obtain

$$
Z(\zeta)=\left[Z\left(\zeta^{*}\right)\right]^{*}+2 i \pi f(\zeta), \quad \Im(\zeta)<0
$$

For real line, $\Im(\zeta)=0$, we use $W(v)=1$ and $\rho_{n}(v)=$ $(L+i v)^{n} /(L-i v)^{n+1}[8]$, and obtain

$$
\begin{aligned}
g^{+}(z) & =\sum_{n=-\infty}^{\infty} i \operatorname{sgn}(n) a_{n} \rho_{n}(z)+i f(z) \\
& =\sum_{n=0}^{\infty} 2 i a_{n} \rho_{n}(z), \quad \Im(z)=0 .
\end{aligned}
$$

We have avoided the singularity on real line by treating the integrals of the basis functions analytically.

A completed scheme 16] is provided through the combination of 24, 25 and (26), which can support an almost arbitrary smooth distribution function $F(v)$ and $F_{p}(v)=\partial F / \partial v$ as input function.

In numerical calculation, we truncate the summation at a finite point $n=N$. In the practical test for Gaussian input function with $N=32$, the program delivers twelve significant decimal digits 9 , where $L$ is an optimal parameter and is set to $2^{-1 / 4} N^{1 / 2}$ as default.

Another good feature of this approach is that the coefficients $a_{n}$ need only be calculated once for all $z$, making the scheme even faster.

Moreover, an unexpected but interesting feature is that, the input function in the L.H.S. of $\sqrt{18}$ is not necessarily a smooth function. The R.H.S. of (18) can transform the real function $F(v)$ in real line to a smooth analytic complex function in whole complex plane with truncation. This feature can help us calculate several (note: not all) non-smooth input functions directly, such as flat top, incomplete Maxwellian, and slowing down. The validity of this approximation will be verified in Sec III.

\section{VISUALIZATIONS OF GENERALIZED PLASMA DISPERSION FUNCTION}

\section{A. Maxwellian distribution}

First, we compare the results of the usual PDF with Maxwellian distribution as input function.

Fig,2 shows a comparison of our scheme (using $N=$ 32 ) with exact $Z(\zeta)$ function via complex error function in standard numerical library on real axis. We find the errors to be around $10^{-12}$ (not shown in the figure), e.g., $Z(1)=-1.076159013825734+0.652049332173291 i$ in our scheme and $Z(1)=-1.076159013825537+$ $0.652049332173292 i$ via standard library. 

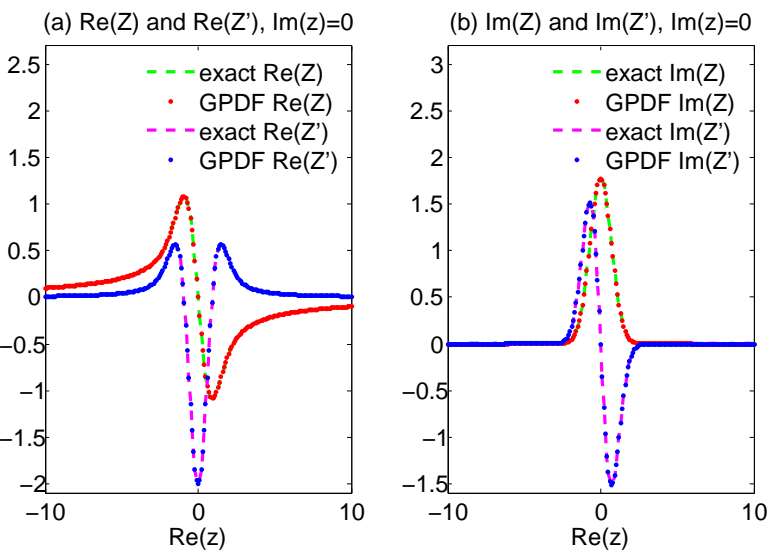

FIG. 2: Comparison of our scheme for GPDF with exact $Z(\zeta)$ function via complex error function in standard numerical library on real axis. (a) $\operatorname{Re}\left(Z^{\prime}\right)$, surface plot

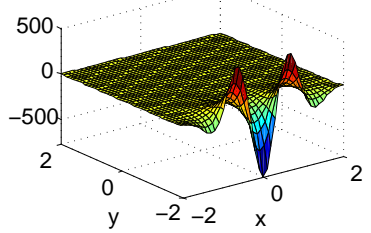

(c) $\operatorname{Re}(Z)$, scales image contour plot

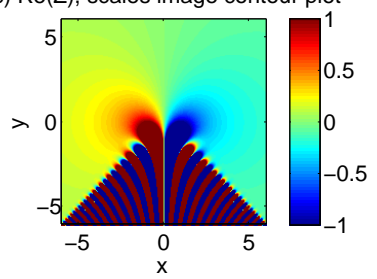

(b) $\operatorname{Im}\left(Z^{\prime}\right)$, surface plot

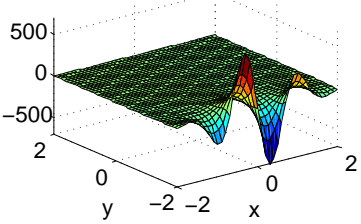

(d) $\operatorname{Im}(Z)$, filled contour plot

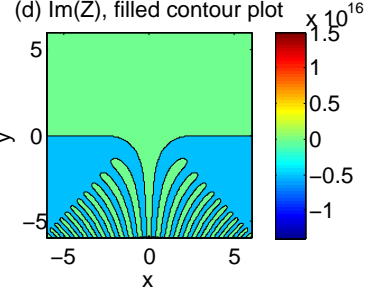

FIG. 3: Visualization of $Z(\zeta)$ and $Z^{\prime}(\zeta)$ with Maxwellian input function.

Fig 3 shows the 2D visualizations of $Z(\zeta)$ and $Z^{\prime}(\zeta)$ produced by our scheme, which shows that the functions are indeed analytically smooth. If we exclude the step for analytic continuation, we will find a jump at real line $\Im(z)=0$ (not shown here).

\section{B. $\kappa$ distribution}

With $F=\frac{1}{\pi\left(v^{2}+1\right)}$ as input function, a result is shown in Fig 4, where we can find a singularity at $z=-i$, which is consistent with analytical result (17).

However, another artificial singular point at $z=i$ [see panel (a)] can be found, where the code yields $N a N$. The two first-order singular points $v-i a=0$ and $v-z=0$ transformed into one second-order singular point $(v-$ $i a)^{2}=0$ when $z=i a$ for Lorentzian input function. An extra approximation $Z\left(\zeta_{0}\right) \simeq Z\left(\zeta_{0}+\epsilon\right)$ in the code is used to treat this kind of singular point, with $\zeta_{0}$ as the (a) $\operatorname{Re}\left(Z^{\prime}\right)$, surface plot

(b) $\operatorname{Im}\left(Z^{\prime}\right)$, surface plot
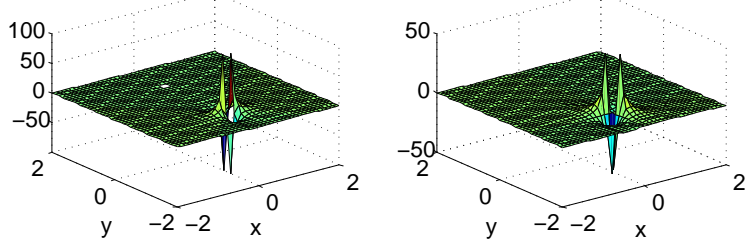

(c) $\operatorname{Re}(Z)$, scales image contour plot
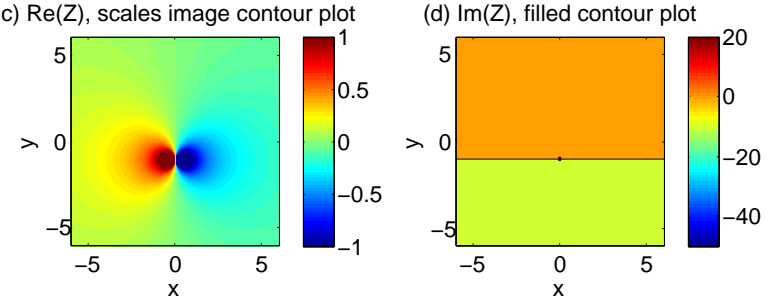

FIG. 4: Visualization of $Z(\zeta)$ and $Z^{\prime}(\zeta)$ with input function $F=\frac{1}{\pi\left(v^{2}+1\right)}$, where artificial singular point $N a N$ at $\zeta=i$ is kept.
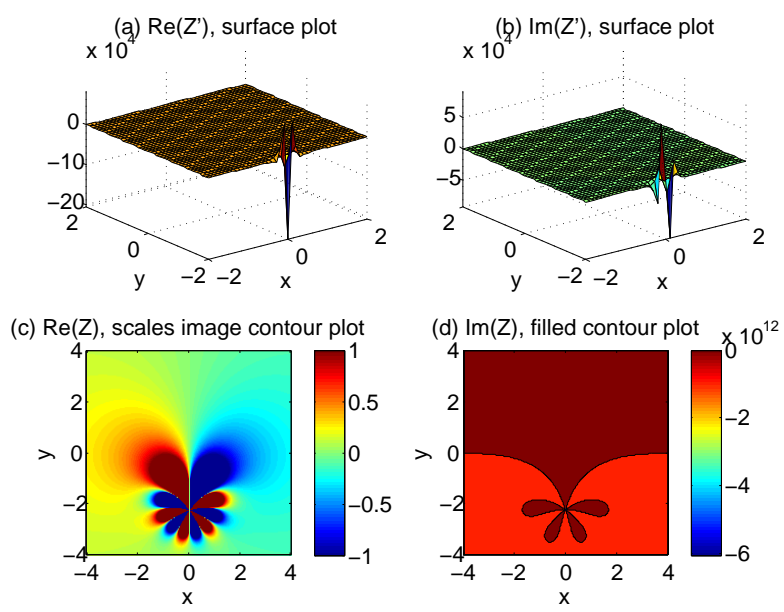

FIG. 5: Visualization of $Z(\zeta)$ and $Z^{\prime}(\zeta)$ with input function $F_{\kappa=5}$.

artificial singular point and $\epsilon$ be a small number, e.g., $10^{-10}$. After the extra treatment, we find GPDF yields exactly the same values as $\pi \times(17)$ in all computation grids (not shown here) with controllable small errors.

After fixing this problem, a result is shown in Fig 5 for $\kappa=5, v_{t}=1$.

To keep $\kappa=1$ the usual Lorentzian distribution, the definition of $\kappa$-distribution in (6) slightly differs from the usual one 4 but is close to the one by Valentini and D'Agosta [3].

\section{C. $\delta$ distributions}

The scheme described in Sec II cannot support several non-standard distribution functions directly, particularly, $\delta$ distribution, which has been widely used for modeling 
(a) $\operatorname{Re}\left(Z^{\prime}\right)$, surface plot

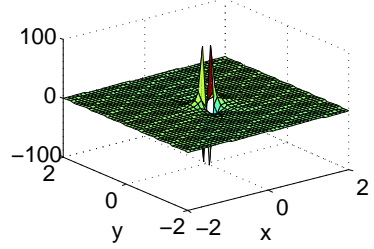

(c) $\operatorname{Re}(Z)$, scales image contour plot

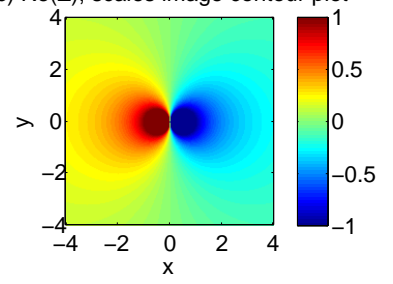

(b) $\operatorname{Im}\left(Z^{\prime}\right)$, surface plot

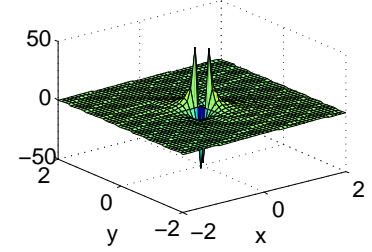

(d) $\operatorname{Im}(Z)$, filled contour plot

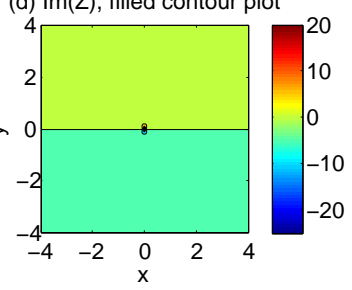

FIG. 6: Visualizations of $Z(\zeta)$ with input function $F_{\delta}$ and parameters $z_{d}=0$.

cold plasma. We treat it separately in the code, via analytical expression.

For $\delta$-distribution $F_{\delta}=\delta\left(z-z_{d}\right)$,

$$
\begin{aligned}
Z(\zeta) & =-\frac{1}{\zeta-z_{d}}, \\
Z_{p}(\zeta) & =\frac{1}{\left(\zeta-z_{d}\right)^{2}},
\end{aligned}
$$

which can also be obtained from (17) using the limit $a \rightarrow$ 0 because $\delta(v)=\lim _{a \rightarrow 0} \frac{1}{\pi} \frac{a}{v^{2}+a^{2}}$. The result is shown in Fig 6 .

\section{Incomplete Maxwellian distributions}

The input function is

$$
F_{\mathrm{IM}}(v)=H(v-\nu) \frac{1}{\sqrt{\pi}} e^{-v^{2}},
$$

which has been investigated comprehensively by Baalrud [5], where $H$ is the Heaviside step function.

As previously mentioned, the numerical scheme cannot treat the $\delta$ function directly. For $Z^{\prime}$, an extra correction term should be added, i.e., $Z_{\mathrm{IM}}^{\prime}(\zeta)=Z_{\mathrm{IM} 0}^{\prime}(\zeta)-$ $\frac{1}{\sqrt{\pi}} e^{-\nu^{2}} /(\zeta-\nu)$, where $Z_{\mathrm{IM} 0}^{\prime}$ is the result without correction.

Fig 7 shows the results of $Z_{\mathrm{IM}}(\zeta)$ and $Z_{\mathrm{IM} 0}^{\prime}(\zeta)$ calculated from our GPDF scheme, with $\nu=1, N=1024$ and $L=10$. Fig 7(a) is in favor of Fig.3(b) by Baalrud[5], which was calculated from a direct numerical integral. Our one-solve-all scheme can solve the same problem at least ten times faster than the direct numerical integral usually with the same accuracy.

A slight error can be found around the real line when $N$ is small, say $N=64$, which arises from the error of Fourier expansion around the sharp step place (Gibbs phenomenon) that requires a large $N$ to overcome. $\begin{array}{ll}\text { (a) Absolute value and argument of } Z_{\mathrm{IM}}(1, \zeta) & \text { (b) Plot of } Z^{\prime}{ }_{\mathrm{IMO}}(1, \zeta)\end{array}$
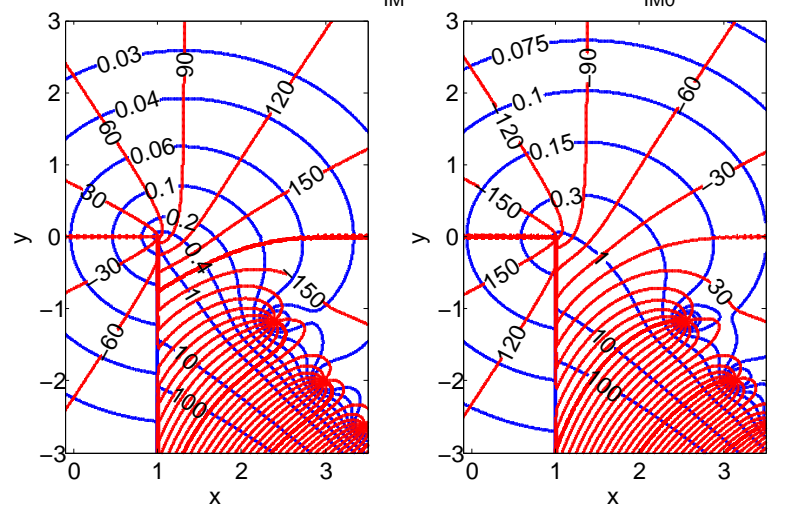

FIG. 7: Visualization of $Z_{\mathrm{IM}}(\zeta)$ and $Z_{\mathrm{IM} 0}^{\prime}(\zeta)$ with input function (28), where the absolute value and argument are calculated using $Z=|Z| e^{i \theta}$. (a) $\operatorname{Re}(Z)$ and $\operatorname{Im}(Z), \operatorname{Re}(\mathrm{z})=0.5$

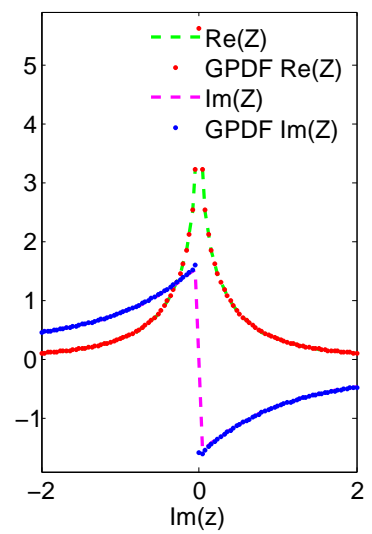

(b) $\operatorname{Re}(Z)$ and $\operatorname{Im}(Z), \operatorname{Im}(\mathrm{z})=-0.1$

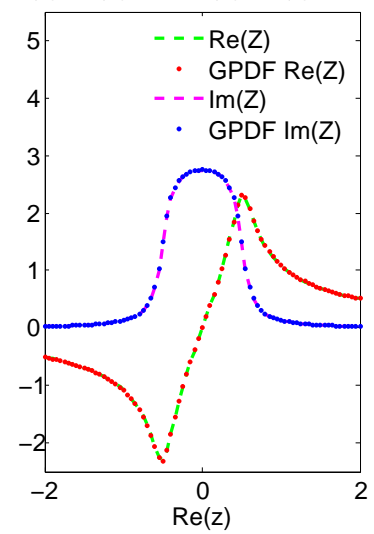

FIG. 8: Comparison of GPDF results and analytical results for $Z(\zeta)$ with input function $F_{\text {Rect }}$ and parameters $z_{a}=-0.5$ and $z_{b}=0.5$.

\section{E. Flat top distribution}

For flat top distribution $F_{\text {Rect }}=\frac{H\left(z-z_{a}\right)-H\left(z-z_{b}\right)}{z_{b}-z_{a}}$,

$$
\begin{aligned}
Z(\zeta) & =\frac{1}{z_{b}-z_{a}}\left[\ln \left(\zeta-z_{b}\right)-\ln \left(\zeta-z_{a}\right)\right] \\
Z_{p}(\zeta) & =\frac{1}{z_{b}-z_{a}}\left[\frac{1}{\zeta-z_{b}}-\frac{1}{\zeta-z_{a}}\right]
\end{aligned}
$$

GPDF results and analytical results are compared in Fig.8. This benchmark indicates that our treatment for GPDF is indeed suitable for both smooth and nonsmooth input function. However, to keep the result exactly the same as (29) at range $z_{a} \leq \zeta \leq z_{b}$ for lower half plane $[\Im(\zeta)<0]$, the analytic continuation term $2 i f(z)$ is set to zero, instead of $2 i /\left(z_{b}-z_{a}\right)$.

For $Z_{\text {Rect }}^{\prime}$, we need also an extra treatment because of the $\delta$ function. 
(a) $\operatorname{Re}\left(Z^{\prime}\right)$, surface plot

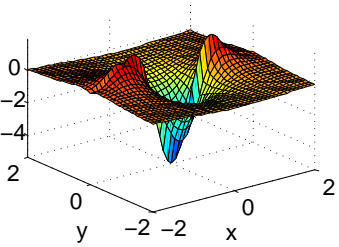

(c) $\operatorname{Re}(Z)$, scales image contour plot

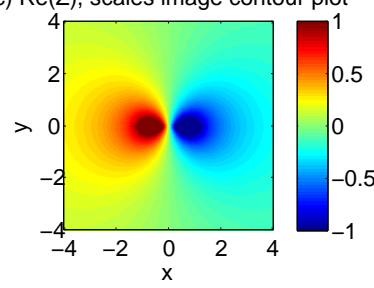

(b) $\operatorname{Im}\left(Z^{\prime}\right)$, surface plot

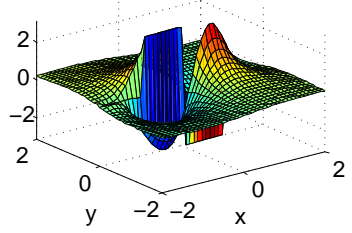

(d) $\operatorname{Im}(Z)$, filled contour plot

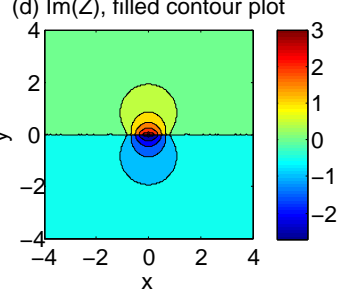

FIG. 9: Visualization of $Z(\zeta)$ and $Z^{\prime}(\zeta)$ with input function $F_{\text {Tri }}$ and parameters $z_{a}=-1.0, z_{b}=0$ and $z_{c}=1$.

\section{F. Triangular distribution}

The distribution function is

$$
\begin{aligned}
F_{\text {Tri }} & =\frac{H\left(z-z_{a}\right)-H\left(z-z_{b}\right)}{\left(z_{b}-z_{a}\right)} \frac{2}{z_{c}-z_{a}}\left(z-z_{a}\right) \\
& -\frac{H\left(z-z_{b}\right)-H\left(z-z_{c}\right)}{\left(z_{c}-z_{b}\right)} \frac{2}{z_{c}-z_{a}}\left(z-z_{c}\right),
\end{aligned}
$$

and corresponding $Z(\zeta)$ is shown in Fig 9 .

The analytic continuation term $2 i f(z)$ for lower half plane $[\Im(\zeta)<0]$ is set to zero. Comparing root finding results and simulation results of Langmuir wave using the methods discussed in Sec IV, we find that the solutions are the same (not shown here) for both cases, i.e., setting the term to zero or non-zero, and agreed with the simulations.

\section{G. Slowing down distributions}

The distribution function is very common in fusion plasma, such as tokamak, for fast particles,

$$
F_{\mathrm{SD}}=\frac{3 \sqrt{3} v_{t}^{2}}{4 \pi} \frac{1}{|v|^{3}+v_{t}^{3}} H\left(v_{c}-|v|\right) .
$$

\section{A result is shown in Fig. 10 .}

One should note that the absolute value of $v$ in $F$ could cause problems in the complex plane, as $\mid \Re(v)+$ $i \Im(v)\left|=\sqrt{\Re(v)^{2}+\Im(v)^{2}} \neq\right| \Re(v) \mid+i \Im(v)$. We use $H(v)$ to rewrite $|v|$ in the code, where $H(\Re(v)+i \Im(v))=$ $H(\Re(v))[\Re(v)+i \Im(v)]$. Hence, we can still use if $(z)$ directly for the analytic continuation term to reduce numerical errors, instead of using the expansion expression $\sum a_{n} \rho_{n}$.

(a) $\operatorname{Re}\left(Z^{\prime}\right)$, surface plot

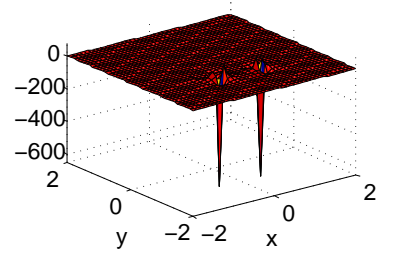

(c) $\operatorname{Re}(Z)$, scales image contour plot

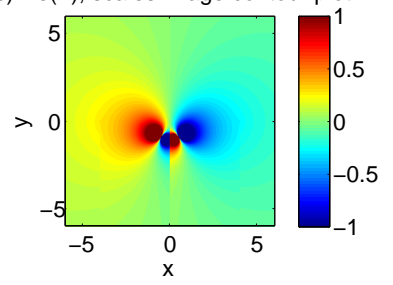

(b) $\operatorname{Im}\left(Z^{\prime}\right)$, surface plot

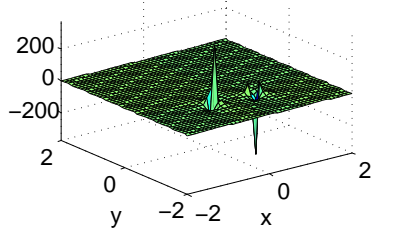

(d) $\operatorname{Im}(Z)$, filled contour plot

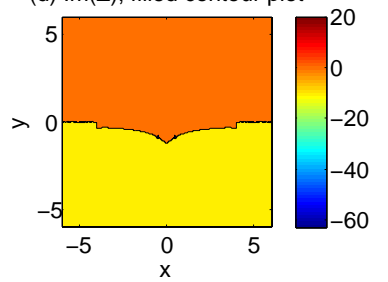

FIG. 10: Visualization of $Z(\zeta)$ and $Z^{\prime}(\zeta)$ with input function $F_{\mathrm{SD}}$ for $v_{t}=1$ and $v_{c}=4$.

(a) $\operatorname{Re}\left(Z^{\prime}\right)$, surface plot

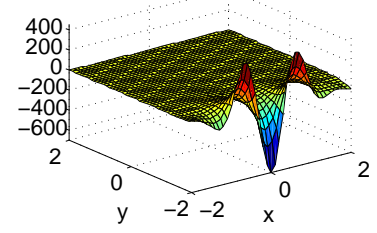

(c) $\operatorname{Re}(Z)$, scales image contour plot

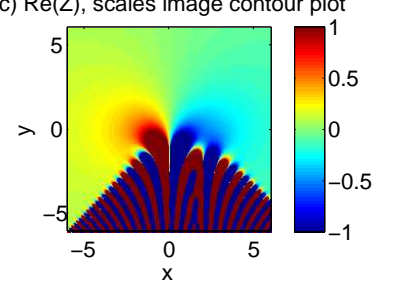

(b) $\operatorname{Im}\left(Z^{\prime}\right)$, surface plot

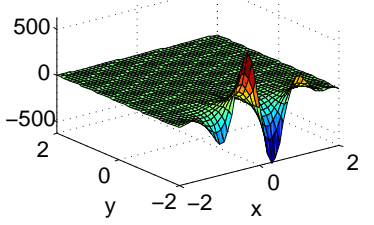

(d) $\operatorname{Im}(Z)$, filled contour plot $\times 10^{16}$

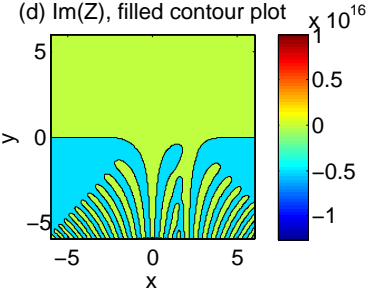

FIG. 11: Visualization of $Z(\zeta)$ and $Z^{\prime}(\zeta)$ with bump-on-tail input function.

\section{H. Other distributions}

The GPDF has several good features such as for the bump-on-tail problem, when using usual PDF, the core plasma and beam plasma should to be treated separately. We can treat it using one input function directly when using GPDF. A result is shown in Fig 11 for $F=0.9 e^{-v^{2}} / \sqrt{\pi}+0.1 e^{-(v-2)^{2}} / \sqrt{\pi}$. A comparison with Fig 3 shows the beam tail affects $Z(\zeta)$ apparently, particularly at the place around $\Re(\zeta)=v_{d}=2$.

\section{Short summary}

The one-solve-all scheme has been shown to be effective. However, to treat non-smooth/analytical input functions, extra corrections should be noted. For nonsmooth flat top and triangular distributions, the method 
of determining the analytic continuation requires further investigation, because it cannot be distinguished by Langmuir wave simulation.

From the visualizations of $Z(\zeta)$ and $Z^{\prime}(\zeta)$ for different types of input functions, the quantitative value, shape, or topology of $Z$ and $Z^{\prime}$ vary considerably, which will then bring different kinetic effects, e.g., Landau damping rate.

\section{DISTRIBUTION FUNCTION EFFECTS ON LANDAU DAMPING}

\section{A. Benchmark GPDF using initial value scheme}

For the initial value scheme, the starting equations are the normalized linear Vlasov-Poisson equations

$$
\left\{\begin{array}{l}
\partial_{t} \delta f=-i k v \delta f+\delta E \partial_{v} f_{0} \\
i k \delta E=-\int \delta f d v
\end{array}\right.
$$

We usually set $\lambda_{D}=1$, then $v_{t}=\sqrt{2}$ in the initial distribution function $f_{0}$, e.g., $f_{0}=\exp \left(-v^{2} / 2\right) / \sqrt{2 \pi}$ for Maxwellian.

Eqs. 32 can be solved as an initial value problem (IVP), e.g., using a 4th-order Runge-Kutta scheme, which should produce the exact linear Landau damping when the Case-Van Kampen mode and numerical errors are ignored[12]. This simulation approach can be a simple and/or final benchmark for GPDF. Disagreements would mean the GPDF has been treated incorrectly. However, the IVP approach is not general, because of the numerical errors from discrete of $v$ and $t$, especially when the phase velocity $v_{p}=\omega / k$ or damping rate are large. A similar but more complicated IVP approach is used by Valentini and D'Agosta 3 . Particle (e.g., particle-in-cell) simulation can also be used, which was also used by Godfrey et al. [14. Particle method can also easily support non-smooth distribution, but is limited by the noise resulting in unfavorable errors. Thus, this technique is not accurate when compared with the above continuum method.

Landau damping of Maxwellian distribution using this continuum IVP approach is verified in a previous work 12 .

We check the Lorentzian distribution to show that our scheme for GPDF with non-Maxwellian distributions is also correct. Fig 12 shows the comparison of Lorentzian distribution Landau damping using IVP scheme and GPDF for $k=0.15$ and $v_{t}=\sqrt{2}$. We find that the simulation and numerical/analytical solutions match very well for both real frequency and damping rate, i.e., $\omega=1.00-0.212 i$.

Another possible numerical approach for 32 is to treat it as an eigenvalue problem. However, the (Landau) damping normal mode is not eigenmode in this system, as discussed by numerous authors (see e.g., 12 and references in). Thus, this approach does not work.
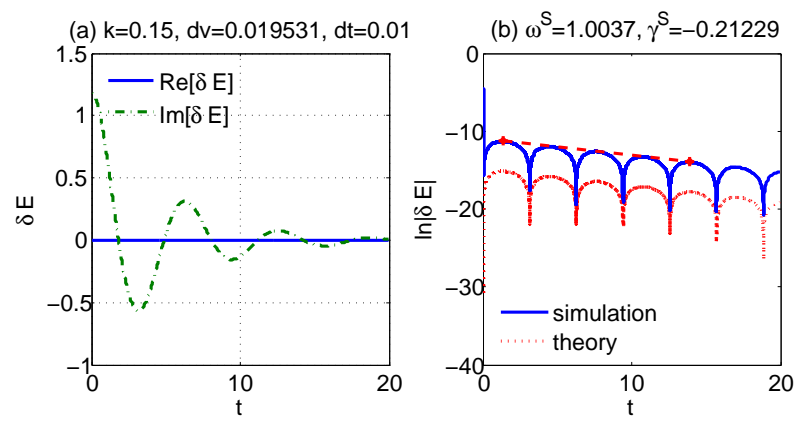

FIG. 12: Benchmark GPDF using initial value simulation for Lorentzian distribution function. The result of root finding for this case is $\omega=1.0000-0.2121 i$.

Notably, for GPDF, (5) would no longer be hold. Thus, $Z_{p}$ should be used for root finding instead of $Z$ to (4) or (11).

\section{B. Effects of discontinuity point}

The $\delta$ function can be modeled as cold plasma, which provides the dispersion relation $\left(\omega-k v_{d}\right)^{2}=\omega_{p}^{2}$. For discontinuity point such as the cases we meet in incomplete Maxwellian or flat top distributions, (29) can be used to solve the flat top case easily, which gives $\left(\omega-k v_{d}\right)^{2}=\omega_{p}^{2}+k^{2} v_{t}^{2}$, where $v_{t}=\left(z_{b}-z_{a}\right) / 2$ and $v_{d}=\left(z_{b}+z_{a}\right) / 2$. The dispersion relation solutions of $\delta$ and flat top distributions are verified by PIC simulation (not shown here). For instance, for flat top distribution $v_{t}=1.0, v_{d}=0, k=1.0$, we obtain $\omega=1.414$, whereas PIC simulation yields $\omega_{r} \simeq 1.40$. For the continuum IVP simulation of discontinuity point, we use the approximation $\partial f_{0} / \partial v=\left[f_{0}\left(v_{0}^{+}\right)-f_{0}\left(v_{0}^{-}\right)\right] / \Delta v$, where $\Delta v$ is the velocity space grid size. In a practical test, the simulation also yields the same result, but is more accurate and has lower noise than PIC simulation. Thus, we use continuum IVP to verify GPDF results.

For incomplete Maxwellian distribution, we can compare the dispersion relation solutions of $Z_{\mathrm{IM}}^{\prime}(\zeta)$ and $Z_{\text {IM0 }}^{\prime}(\zeta)$ to investigate the effects of discontinuity point.

The results are shown in Fig 13 , with $\nu=-0.1 v_{t}$ and $v_{t}=\sqrt{2}$. For example, $k=1.0, Z_{\mathrm{IM}}^{\prime}$ yields $\omega^{+}=2.0409-\mathrm{i} 0.8801$ and $\omega^{-}=-0.4542+\mathrm{i} 3.5173 \mathrm{E}-5 ; Z_{\mathrm{IM} 0}^{\prime}$ yields $\omega=2.0843$-i0.7871, whereas IVP simulation yields $\omega \simeq 0.454+\mathrm{i} 0$. Our solutions of $\omega^{ \pm}$via GPDF are in favor of the solutions by Baalrud [5]. This benchmark provides further verification of the one-solve-all scheme.

Fig 13 shows that the discontinuity point at $v=\nu$ changes the dispersion properties considerably, for instance, a new nearly undamped branch can be found, which should be caused by the lack of resonance particles at $v_{p}=\omega / k<\nu$. The differences between the solutions of $Z_{\mathrm{IM}}^{\prime}(\zeta)$ and $Z_{\mathrm{IM} 0}^{\prime}(\zeta)$ also indicates that an incorrect treatment of discontinuity point will yield inaccurate results. 

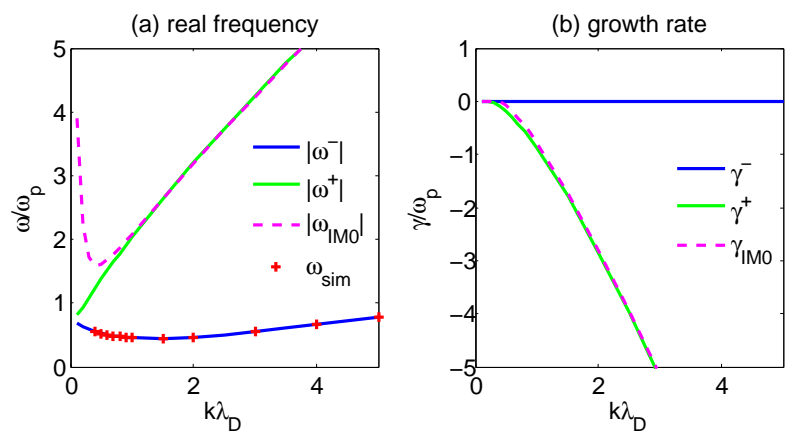

FIG. 13: Dispersion relation solutions (root finding) of incomplete Maxwellian distribution with $\nu=-0.1 v_{t}$, which agree with the simulation results very well.
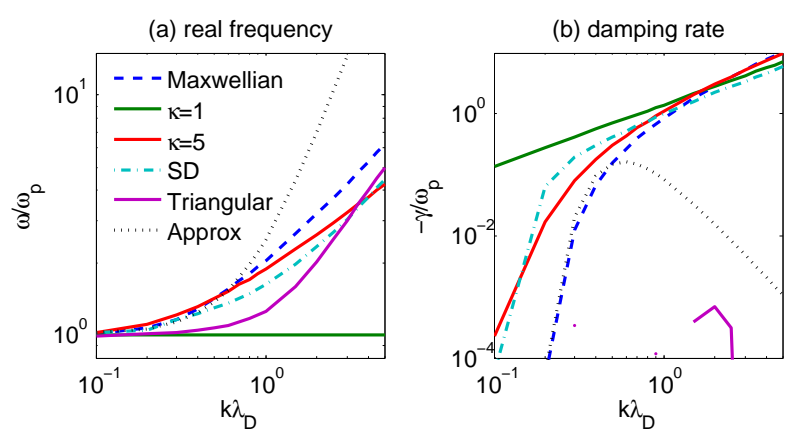

FIG. 14: Distribution function effects on Landau damping with different initial distributions, where the approximate solution by (33) for Maxwellian distribution is also shown.

\section{Results of distribution function effects}

We use GPDF to revisit the distribution function effects on Landau damping.

For Maxwellian distribution and small $k$, approximate analytical expressions for real frequency and growth rate are 1 ]

$$
\begin{gathered}
\omega_{r}^{2}=\omega_{p}^{2}\left(1+3 k^{2} \lambda_{D}^{2}\right), \\
\omega_{i}=\left.\frac{\pi}{2} \frac{\omega_{r}^{3}}{k^{2}} \frac{\partial f_{0}}{\partial v}\right|_{v=\omega_{r} / k} .
\end{gathered}
$$

The effects of $\kappa$-distribution on Landau damping, particularly for space plasma, are discussed in detail by Thorne and Summers 13. The incomplete Maxwellian distribution is discussed by Baalrud [5].

We choose Maxwellian, $\kappa$, and slowing down distributions with $v_{t}=\sqrt{2}$ as well as triangular distributions with the same parameters as in Fig 9 for further comparisons. Fig 14 shows the results of $\omega_{r}$ vs. $k$ and $\gamma$ vs. $k$. For triangular distribution, a non-zero $\gamma$ around $\pm 10^{-4}$ exists but is sensitive to initial guessing for the root find- ing, which should be caused by the jump of $Z^{\prime}$ around $\Im(z)=0$ as shown in Fig. 9 .

Table I shows the quantitative value of the solutions, where $\omega^{G}$ is solved from GPDF and $\omega^{S}$ is from IVP sim-

TABLE I: Comparison of the Langmuir wave solutions with different distribution functions and $k=1.0$.

\begin{tabular}{ccccc}
\hline \hline- & $\omega_{r}^{G}$ & $\omega_{i}^{G}$ & $\omega_{r}^{S}$ & $\omega_{i}^{S}$ \\
\hline Maxwellian & 2.0459 & -0.8513 & 2.01 & -0.85 \\
$\kappa=1$ & 1.0000 & -1.4142 & 1.00 & -1.40 \\
$\kappa=5$ & 1.8786 & -1.0866 & 1.82 & -1.08 \\
Slowing down & 1.6240 & -0.9975 & $\simeq 1.68$ & $\simeq-0.87$ \\
Triangular & 1.2577 & $4.2 \mathrm{E}-4$ & $\simeq 1.26$ & $\simeq 0.005$ \\
\hline \hline
\end{tabular}

ulation. For non-smooth input distributions, the simulation is not robust and is sensitive to parameters and initial conditions. Several results in table $(k=1.0)$ are very rough (labeled with ' $\simeq$ '). For instance, the error of the result in this table for slowing down distribution is very large (approximately 10\%), whereas, for small $k$ and small damping rate, e.g., $k=0.5$, IVP simulation is more robust and accurate, and we obtain $\omega_{S D}^{S}=1.29-0.42 i$ compared with $\omega_{S D}^{G}=1.2876-0.4119 i$.

\section{SUMMARY AND DISCUSSION}

The analytical properties and one-solve-all numerical scheme for generalized plasma dispersion function, which provides a useful tool for treating linear effects of almost arbitrary distribution functions, are discussed. The exact distribution function effects on Landau damping are revisited to demonstrate an application.

The one-solve-all scheme can also be used analytically as an expansion method for GPDF, in addition to the usual Taylor expansion scheme used.

Our method cannot be used directly for relativistic 14, 15. or other more complicated dispersion functions because those dispersion functions are usually not in HT form. However, similar orthogonal functions expansion treatment may be used, as mentioned by Robinson [7.

\section{ACKNOWLEDGEMENTS}

Discussions with Y. R. Lin-Liu at the early stage of this project to understand the treatment of usual PDF are appreciated. This work is support by the NSF of China under Grants No.11235009, the ITER-CN under Grant No. 2013GB104004 and Fundamental Research Fund for Chinese Central Universities. 
[1] D. R. Nicholson, Introduction to Plasma Theory (Wiley, 1983).

[2] B. D. Fried and S. D. Conte, The Plasma Dispersion Function - THE HILBERT TRANSFORM OF THE GAUSSIAN (Academic Press, New York and London, 1961). Erratum: Math. Comp. v. 26, 1972, no. 119, p. 814. Reviews and Descriptions of Tables and Books, Math. Comp., v. 17, 1963, pp. 94-95.

[3] F. Valentini and R. D'Agosta, Phys. Plasmas 14, 092111 (2007).

[4] D. Summers and R. M. Thorne, Phys. Fluids B 3, 1835 (1991).

[5] S. D. Baalrud, Phys. Plasmas 20, 012118 (2013).

[6] M. A. Hellberg and R. L. Mace, Phys. Plasmas 9, 1495 (2002).

[7] P. A. Robinson, Journal of Computational Physics 88, 381 (1990).

[8] J. A. C. Weideman, Mathematics of Computation 64, 745 (1995).

[9] J. A. C. Weideman, SIAM J. Numer. Anal. 31, 1497
(1994).

[10] W. D. Jones, H. J. Doucet and J. M. Buzzi, An Introduction to the Linear Theories and Methods of Electrostatic Waves in Plasmas (Springer, 1985).

[11] P. Guio, J. Lilensten, W. Kofman and N. Bjorna, Annales Geophysicae 16, 1226 (1998).

[12] H. S. Xie, Constant residual electrostatic electron plasma mode in Vlasov-Ampere system, submitted to Phys. Plasmas.

[13] R. M. Thorne and D. Summers, Phys. Fluids B 3, 2117 (1991).

[14] B. B. Godfrey, B. S. Newberger and K. A. Taggart, IEEE Transactions on Plasma Science 3, 60 (1975).

[15] F. Castejon and S. S. Pavlov, Phys. Plasmas 13, 072105 (2006).

[16] See supplementary material at [URL will be inserted by AIP] for the MATLAB version numerical routines, where GPDF, root finding, IVP simulation and test plotting codes are included. 